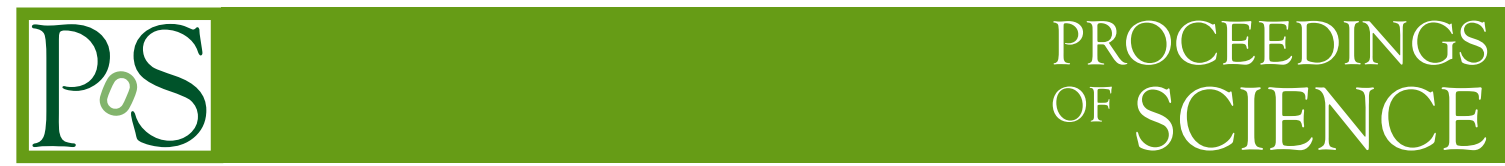

\title{
Highlights from the CMS experiment
}

\author{
Marco Pieri ${ }^{a * *}$ \\ ${ }^{a}$ UC San Diego, United States \\ *on behalf of the CMS collaboration \\ E-mail: marco.pieri@cern.ch
}

The current activities and results from the CMS experiment are reviewed. Some of the most recent results from the analysis of the Run 2 data are presented, including detector related performance studies and physics analyses. The preparation for Run 3 is also addressed and the prospects for the High Luminosity phase of the LHC are briefly outlined.

The European Physical Society Conference on High Energy Physics (EPS-HEP2021), 26-30 July 2021.

Online conference, jointly organized by Universität Hamburg and the research center DESY. 


\section{Introduction}

The CMS Collaboration is currently engaged on several fronts: the completion of the Long Shutdown 2 (LS2), between Run 2 and Run 3, that was also impacted by the difficulties related to the COVID pandemic; the analysis of the large amount of data collected in the Run 2 of the LHC; the preparation for the Run 3, scheduled to start in spring 2022; and the studies and upgrades of the detector in view of the High Luminosity phase of the LHC (HL-LHC), expected to start in 2027, including the Snowmass workshop that will be completed in 2022.

\section{Long shutdown 2 activities}

Since the end of Run 2 in 2018, the LHC and the accelerator and the detectors have undergone the LS2 period and all activities of preparation for the Run 3 have been carried out. The CMS detector [1] has been upgraded in several of its components: the first stations of the Gas Electron Multiplier muon detectors have been installed and this is the first Phase II detectors for HL-LHC that will be used in CMS. The upgrade of the readout of the Hadron calorimeter has been completed, the pixel tracker has been refurbished and the full first layer has been replaced and finally the new beam pipe for Run 3 and HL-LHC has been installed. Several improvements have been made in the trigger, including the development of a hetherogeneous architecture of the High Level Trigger (HLT) which uses CPUs and GPUs.

\section{Run 2 analyses}

The analysis of Run 2 data is still one of the main priorities in CMS and the physics output continues to be very large. CMS presented 26 new preliminary results at the EPS conference of which only a fraction is briefly reported here. Many more and with more details are presented in these proceedings. Most results are based on the Run 2 data during which CMS recorded $150 \mathrm{fb}^{-1}$ at $\sqrt{s}=13 \mathrm{TeV}$ with $140 \mathrm{fb}^{-1}$ usable for analysis. At the time of the conference presentation, CMS published or submitted to a journal 1054 papers based on collision data and continues to submit and publish papers at a rate similar to that of the last few years.

\subsection{Studies of physics objects and other physics related tools}

A crucial aspect of the physics analysis is the continuous study and development of the tools that are needed to obtain the best physics results. The study of physics objects, including the improvement of performance as well as the improvements of the detector simulation which are gauged from the data-Monte Carlo (MC) comparison, is still ongoing with the Run 2 data. This and further studies in view of Run 3 will be instrumental to improve the analysis in Run 3 and should allow to explore new channels and increase the sensitivity beyond the level corresponding to that expected from the increase of integrated luminosity.

\subsubsection{New luminosity analysis of 2015 and 2016 data}

CMS recently completed the luminosity analysis of 2015 and 2016 data, reaching the ultimate precision [2]. The luminosity uncertainty of 2016 data decreased from from $2.5 \%$ in the previous 
analysis [3] to $1.2 \%$ bringing the uncertainty for the full Run 2 data-taking period to $1.6 \%$. Updated luminosity measurements for 2017 and 2018 data are also expected to come soon. The luminosity is measured in CMS with several redundant methods that are compared and cross checked to obtain the optimal performance. Figure 1 shows a sketch of a sector of the CMS detector with the location of the different sub-detectors used for the luminosity measurement and a plot showing the linearity of the number of clusters in the pixel detector versus the pileup (proportional to the instantaneous luminosity) for the nominal luminosity measurement method.

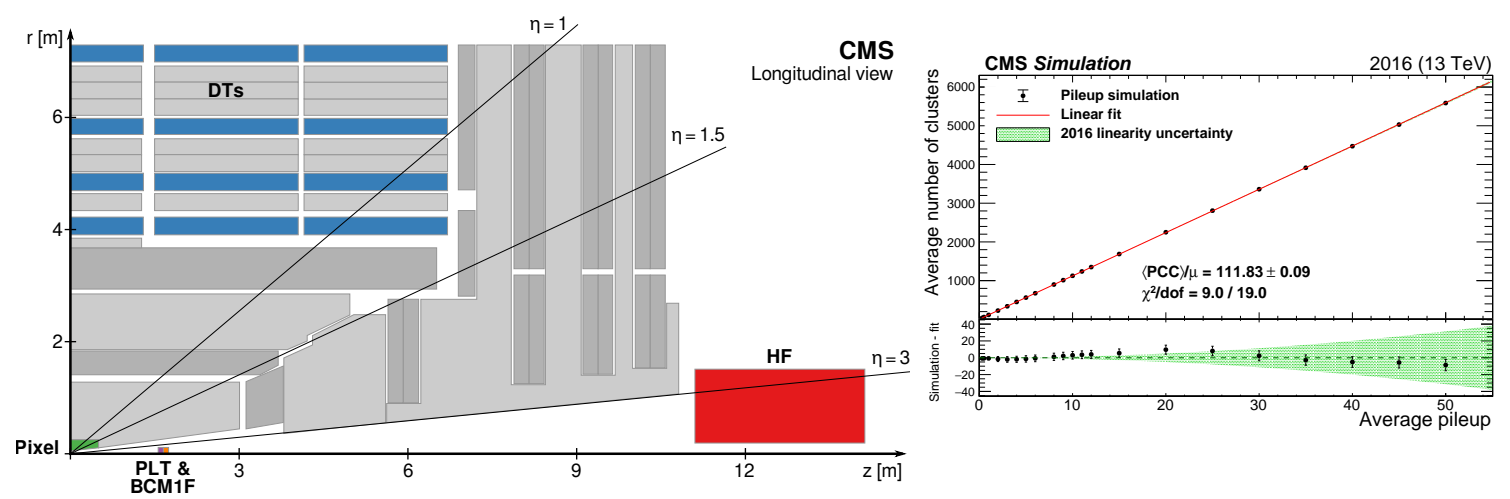

Figure 1: (left) Sketch of a quarter of a longitudinal section of the CMS detector. The main subdetectors used to measure the luminosity in Run 2 are highlighted. (right) Mean number of simulated pixel clusters as a function of the mean pileup. The red curve is a straight line fit. The lower panel shows the difference between the simulation and the linear fit with the green band indicating the linearity uncertainty.

\subsection{Electroweak measurements}

CMS recently performed a measurement of the invisible width of the $\mathrm{Z}$ boson [4]. The measurement of the ratio $\Gamma_{\nu v} / \Gamma_{\ell \ell}$ is carried out with a simultaneous fit of the $P_{t}^{\text {miss }}$ distribution in three categories enriched in $Z \rightarrow v v, Z \rightarrow \mu \mu$, and $Z \rightarrow e e$ decays. $P_{t}^{\text {miss }}$ is the transverse missing momentum computed without taking ito account the momenta of the selected charged leptons in such a way to have similar distributions in the three categories. The distribution of the fitted momenta with the results of the fit are shown in Fig. 2 left. CMS measures $\Gamma_{\text {inv }}=523 \pm 3$ (stat) \pm 16 (syst) MeV. This result is shown in Fig. 2 right together with the corresponding direct measurement from the LEP experiments and the LEP combined one. The CMS uncertainty is smaller than that of the individual LEP experiments and the same as the one of the combined LEP result.

Another very interesting recent result that was presented at this conference for the first time is the first observation of the production of three $\mathrm{J} / \psi$ mesons in proton-proton collisions [5]. Using Run 2 data CMS carried out a search of three $\mathrm{J} / \psi$ decaying into $\mu^{+} \mu^{-}$pairs. This process receives contributions from single (SPS), double (DPS), and triple (TPS) parton scattering as depicted in Fig. 3 left. Five candidate events are selected and this process is observed with a significance larger than $5 \sigma$. The fiducial cross section for this process is found to be $\sigma(\mathrm{pp} \rightarrow \mathrm{J} / \psi \mathrm{J} / \psi \mathrm{J} / \psi \mathrm{X})=$ $272_{-104}^{+141}$ (stat) \pm 17 (syst) fb.

The result is compared to theoretical expectations for the production of three $\mathrm{J} / \psi$ mesons in SPS, DPS, and TPS and the DPS effective cross section parameter is extracted, $\sigma_{\text {eff,DPS }}=$ 

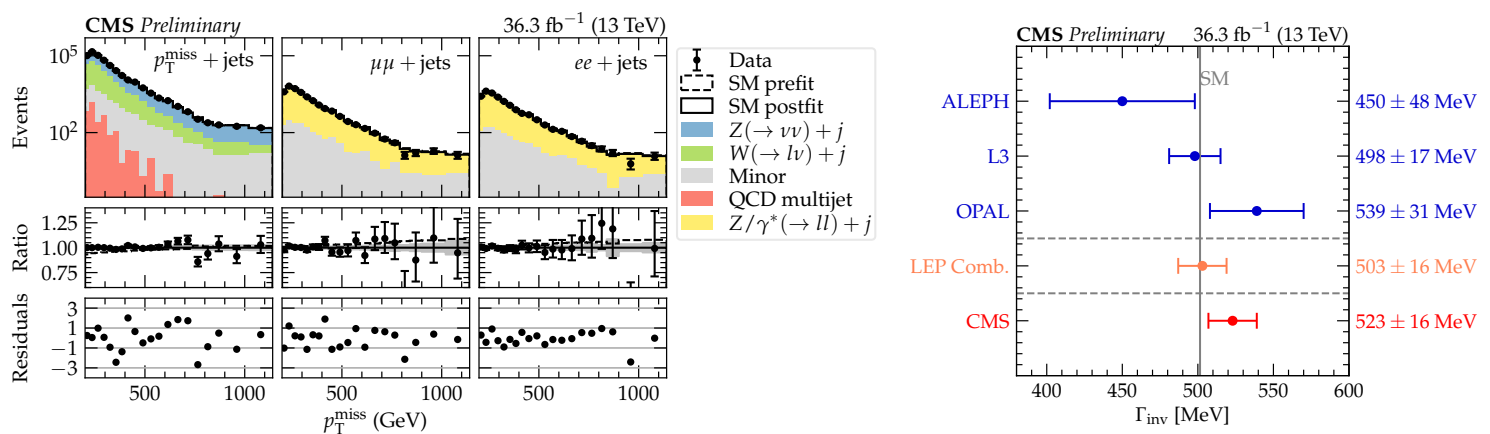

Figure 2: (left) Distribution of $P_{T}^{\text {miss }}$ for the $Z \rightarrow v v, Z \rightarrow \mu \mu$, and $Z \rightarrow e e$ enriched categories. Selected muons and electrons are not considered for the $P_{T}^{\text {miss }}$ calculation. The residuals in the bottom panel are the difference between data and fit results, divided by the uncertainties summed in quadrature. (right) Measurement of the $\mathrm{Z}$ invisible width compared to the LEP direct measurements.
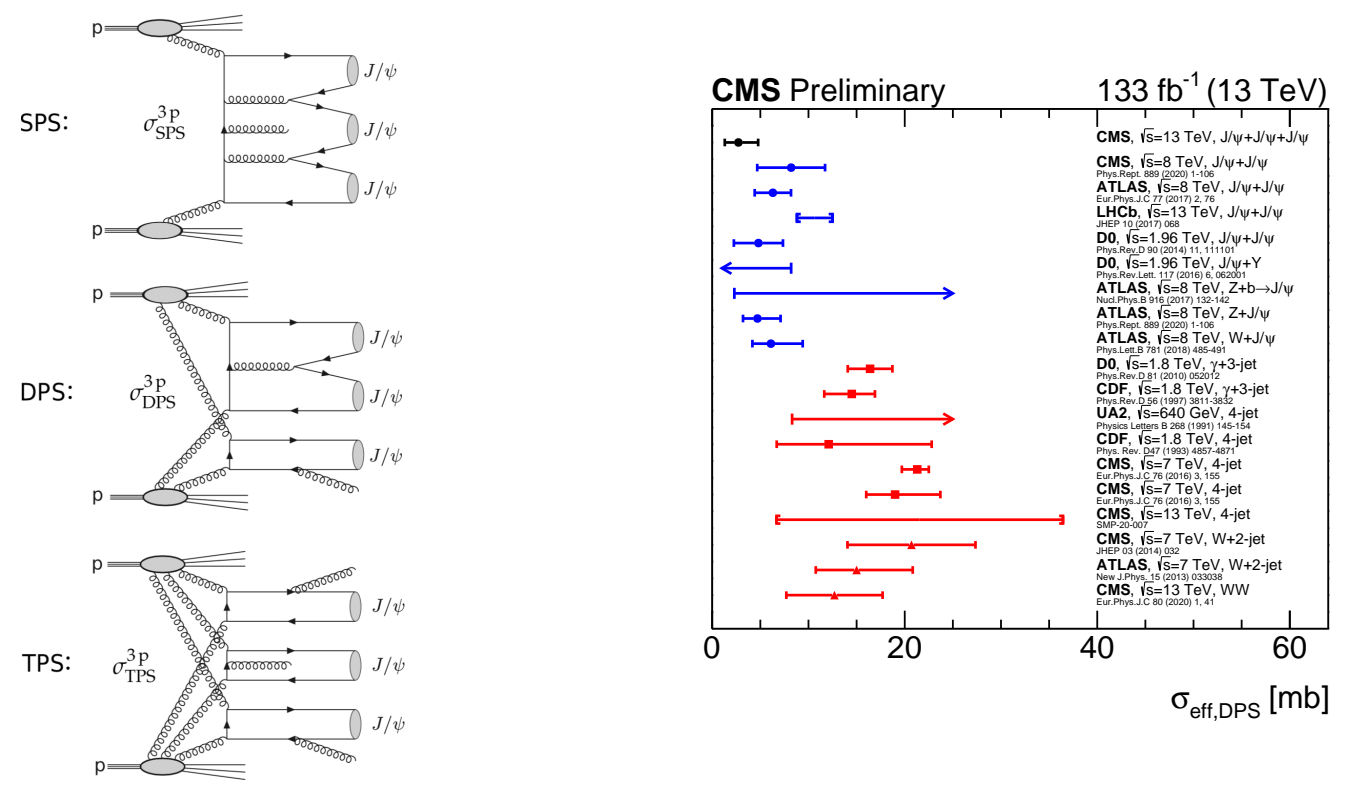

Figure 3: (left) Feynman diagrams for the prompt triple $\mathrm{J} / \psi$ production. (right) Comparison of the extracted effective DPS cross section $\sigma_{\text {eff,DPS }}$ with the same parameter extracted from other measurements.

$2.7_{-1.0}^{+1.4}(\exp )_{-1.0}^{+1.5}$ (theo) $\mathrm{mb}$, and it is compared to previous measurements from several other processes (Fig. 3 right). Triple $\mathrm{J} / \psi$ production is a candidate process for first observation of TPS.

\subsection{Higgs measurements}

Among the recent measurement of the Higgs boson properties, a very relevant one has been the first evidence of the Higgs boson decay into a pair of muons [6] that constitutes also the first evidence of the Higgs boson coupling to second generation fermions. CMS measured this decay with a signal strength relative to the standard model (SM) prediction of $\mu=1.19_{-0.39}^{+0.40}$ (stat) ${ }_{-0.14}^{+0.15}$ (syst) corresponding to a significance of $3.0 \sigma$ ( $2.5 \sigma$ expected $)$. 


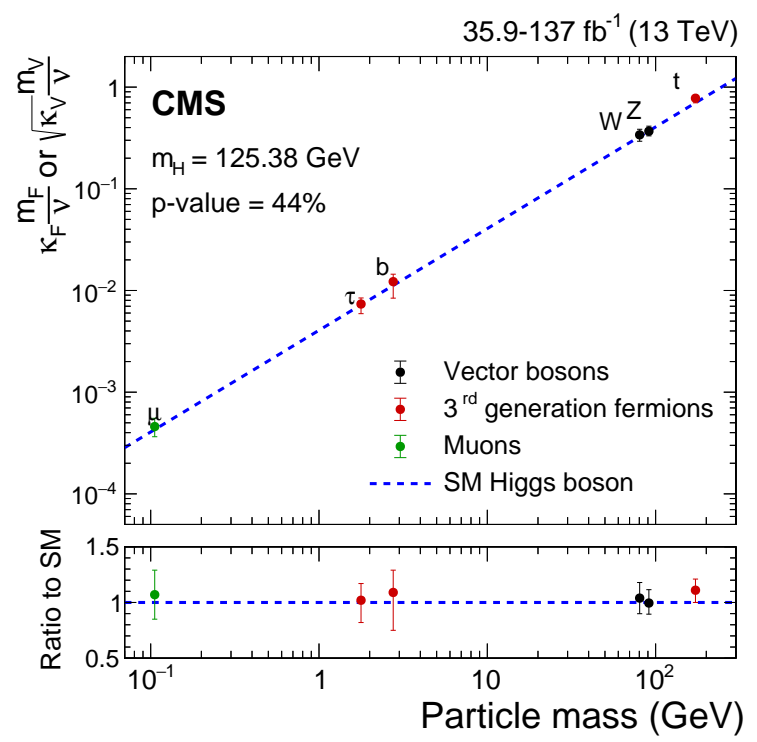

Figure 4: The best fit estimates for the reduced coupling modifiers extracted for fermions and weak bosons in the resolved $\kappa$-framework compared to the corresponding predictions from the SM.

Figure 4 shows the CMS result in terms of the Higgs boson reduced couplings with the various SM particles coupling versus the mass of the particles.
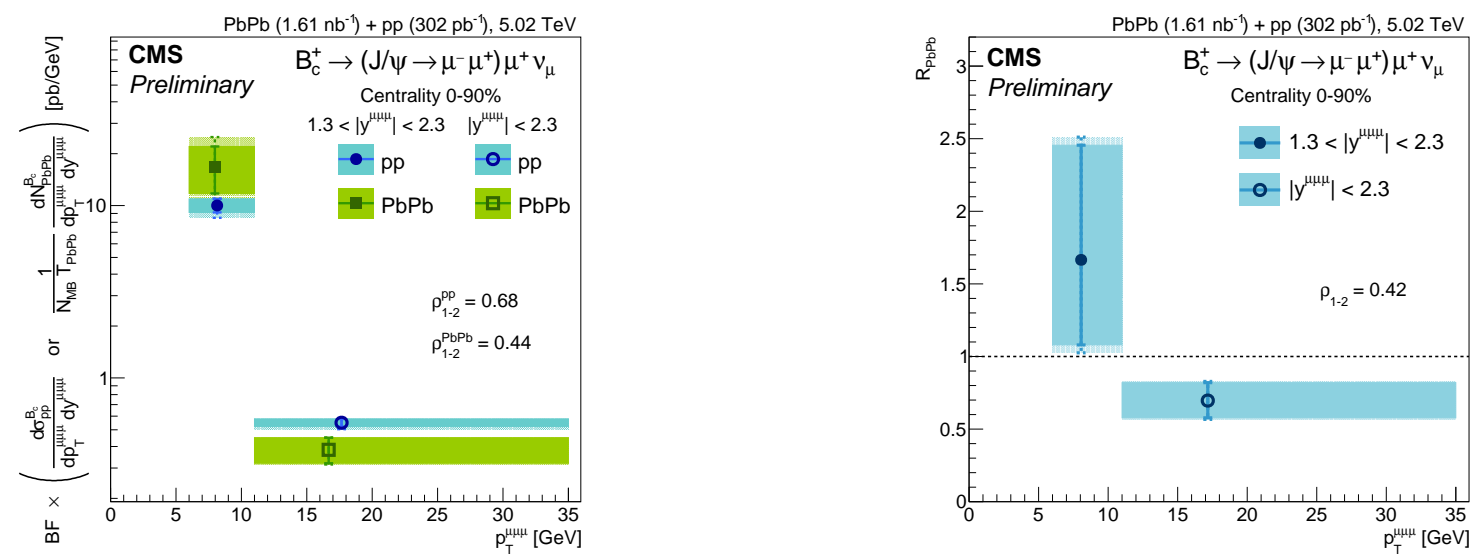

Figure 5: (left) $\mathrm{B}+\mathrm{c}$ meson production pp-equivalent cross-section times branching fraction of the studied decay in $\mathrm{pp}$ and $\mathrm{PbPb}$ collisions. (right) $\mathrm{B}+\mathrm{c}$ meson nuclear modification factor in $p_{T}^{\mu \mu \mu}$ bins corresponding to different rapidity ranges.

\subsection{Heavy Ion results}

$B_{c}^{+}$mesons contains both $\mathrm{b}$ and $\mathrm{c}$ quark and can act as a bridge between bottom and charm mesons, and quarkonia for what concerns interactions with the quark gluon plasma. $B_{c}^{+}$are detected through their decays $B_{c}^{+} \rightarrow \mathrm{J} / \psi \mu v_{\mu}$ by searching for three muons among which there is an opposite pair with mass consistent with a J $/ \psi$. Using the Run 2 data, CMS carried out the first observation of 
the production of $B_{c}^{+}$mesons in $\mathrm{PbPb}$ collisions with a significance above 5 $\sigma$ [7]. The $B_{c}^{+}$process can provides unique insight into the interplay between suppression and recombination in the hot and dense matter created in heavy ion collisions, especially at low $p_{T}$. Figure 5 shows the measurement of the $B_{c}^{+}$production cross section in $\mathrm{pp}$ and $\mathrm{PbPb}$ collisions and their ratio, the nuclear modification factor.

\section{Searches for new physics}

After almost three years since the end of Run 2, most standard searches have been carried out and unfortunately we did not yet find signs of new physics. Most of the remaining analysis being carried out now are aimed at even more exotic signatures and at models which have not yet been covered. An especially active field is the search for Long Lived Particles (LLP) which travel some distance from the interaction point before decaying and give rise to more exotic signatures. They could have escaped detection until now as we concentrated on more standard signatures where the high $p_{T}$ decay products mainly come from the primary interaction point.

Some example of most recent searches are presented in the following.
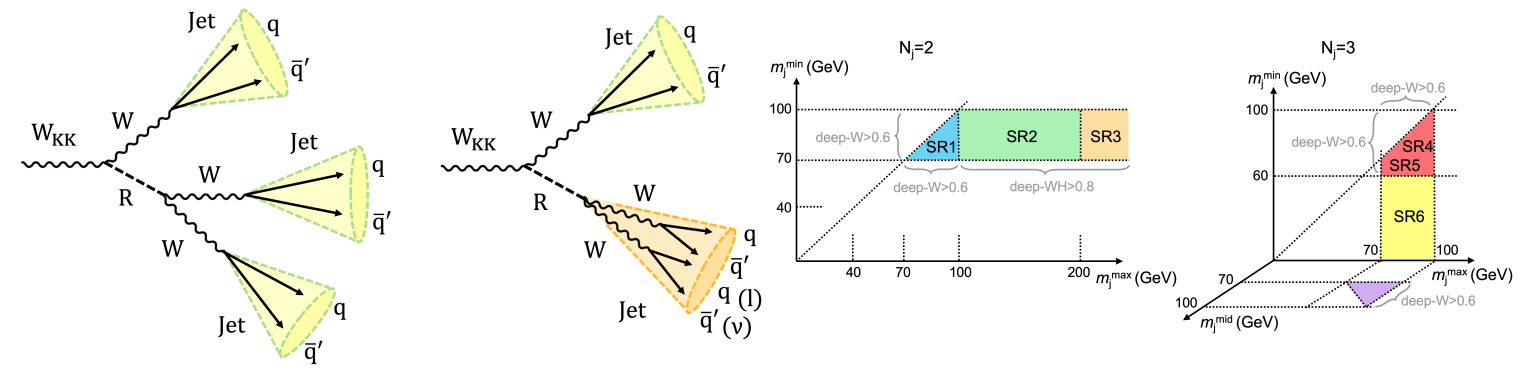

Figure 6: (left two pictures) Schematic diagrams of the decay of $W_{K K}$ to three $\mathrm{W}$ bosons in the resolved and merged scenarios. (right two pictures) Categorization for two- and three-jet events (indicating the defiition of the six signal regions SR1-6, indicated by the coloured areas. The violet area is an additional control region.

\subsection{Search for resonances decaying into three $\mathrm{W}$ bosons}

A search for three $\mathrm{W}$ boson resonances, with fully hadronic $\mathrm{W}$ decays was carried out using the full Run 2 data [8]. A Kaluza-Klein excited massive gauge boson $W_{K K}$ is assumed to decay into a radion $\mathrm{R}$ and a $\mathrm{W}$ boson with the radion decaying into two $\mathrm{W}$ bosons is considered: $X \rightarrow$ $R W \rightarrow W W W$. Each $\mathrm{W}$ boson is reconstructed as a large radius jet while the radion can be merged or resolved when it decays into one or two reconstructed large radius jets. The search is carried out in six signal categories (SR 1-6) based on the number of jets and maximum and minimum reconstructed jet mass. A sketch of the categories is shown in Fig. 6. No statistically significant excess is found and exclusion limits are derived. Results are also combined with the complementary search in the channel where one of the $\mathrm{W}$ decays undergoes a semileptonic decay [9]. Fig. 7 shows the exclusion limits obtained.

\subsection{Search for long lived Heavy Neutral Leptons}

A search for new Heavy Neutral Leptons (HNL) produced through the mixing with SM neutrinos addressing final states with three charged leptons $\ell$ with $\ell=\mathrm{e}$ or $\mu$ and a neutrino is 

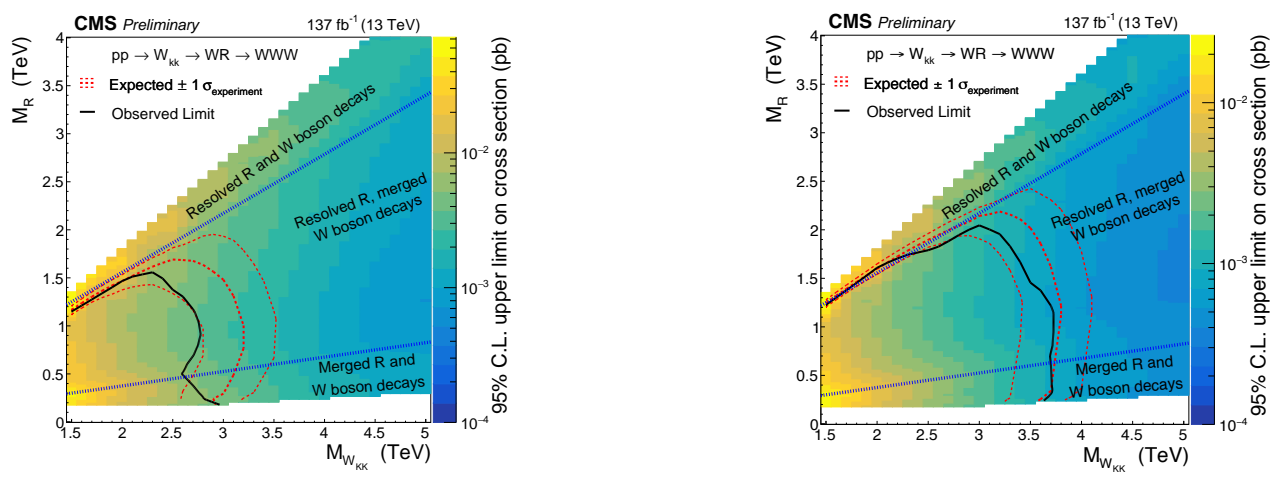

Figure 7: (left) Expected (red dashed lines) and observed (solid black line) upper limits at 95\% CL on the product of the signal cross section and the branching fraction to three $\mathrm{W}$ bosons from the all-hadronic search as functions of the $W_{K K}$ and $\mathrm{R}$ resonance masses. (right) Corresponding results for the combination with the semileptonic channel.

performed [10]. The HNL can decay into $Z v$ or $W \ell$ and can be of Dirac (opposite charge leptons) or Majorana type (leptons can have the same charge). The Feynman diagrams of this process for the Majorana case are shown in Fig. 8. For small values of the HNL mass $(<20 \mathrm{GeV})$ and of the HNL-SM neutrino mixing parameter the HNL may be long lived. CMS searched for three lepton events (e and $\mu$ ), one prompt and the other two forming a displaced secondary vertex. No signal has been observed and and the obtained exclusion limits are shown in Fig. 9.
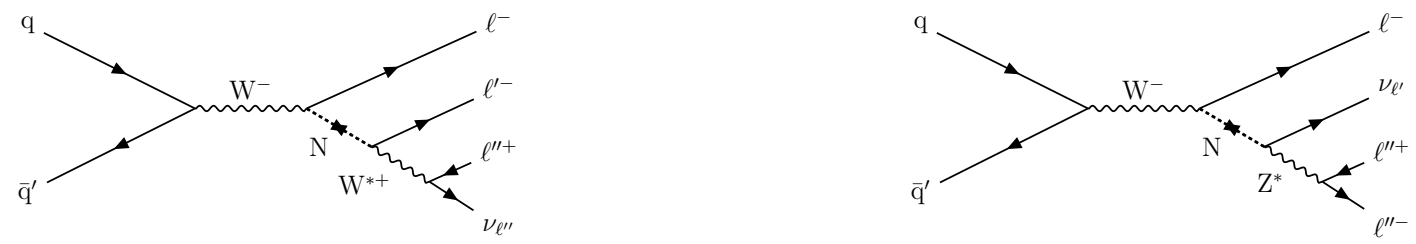

Figure 8: Typical diagrams for the production of an HNL (N) through its mixing with a SM neutrino, leading to final states with three charged leptons and a neutrino. In this case the HNL is assumed to be a Majorana neutrino and its decay is mediated by either a $\mathrm{W}^{*}$ (left) or a $\mathrm{Z}^{*}$ (right) boson.

\subsection{Search for SUSY in HH(4b)+MET final states}

A search for two Higgs bosons, each decaying into a pair of b quarks, accompanied by missing transverse momentum is performed [11]. The search is carried out in two categories based on the angular separation between the two b-jets $\left(\Delta R_{b b}\right)$ : boosted $\left(\Delta R_{b b}<0.8\right)$ and resolved $\left(\Delta R_{b b}>0.4\right)$, the second having the priority. No significant excess over the background expectations is observed and results are interpreted using simplified SUSY models. A $\tilde{\chi}_{1}^{0}$ of mass between 175 and 1025 $\mathrm{GeV}$ and a gluino with mass less than $2330 \mathrm{GeV}$ are excluded at $95 \%$ C.L. assuming two processes: a process $\tilde{\chi}_{1}^{0} \chi_{1}^{0} \rightarrow H H \tilde{G} \tilde{G}$ where the LSP is a gravitino (TChiHH-G model) and the strong production of a pair of gluinos, each of which decays via a three-body process to quarks and a heavy neutralino subsequently decaying to a Higgs boson and a $\tilde{\chi}_{1}^{0}$ (T5HH model). 

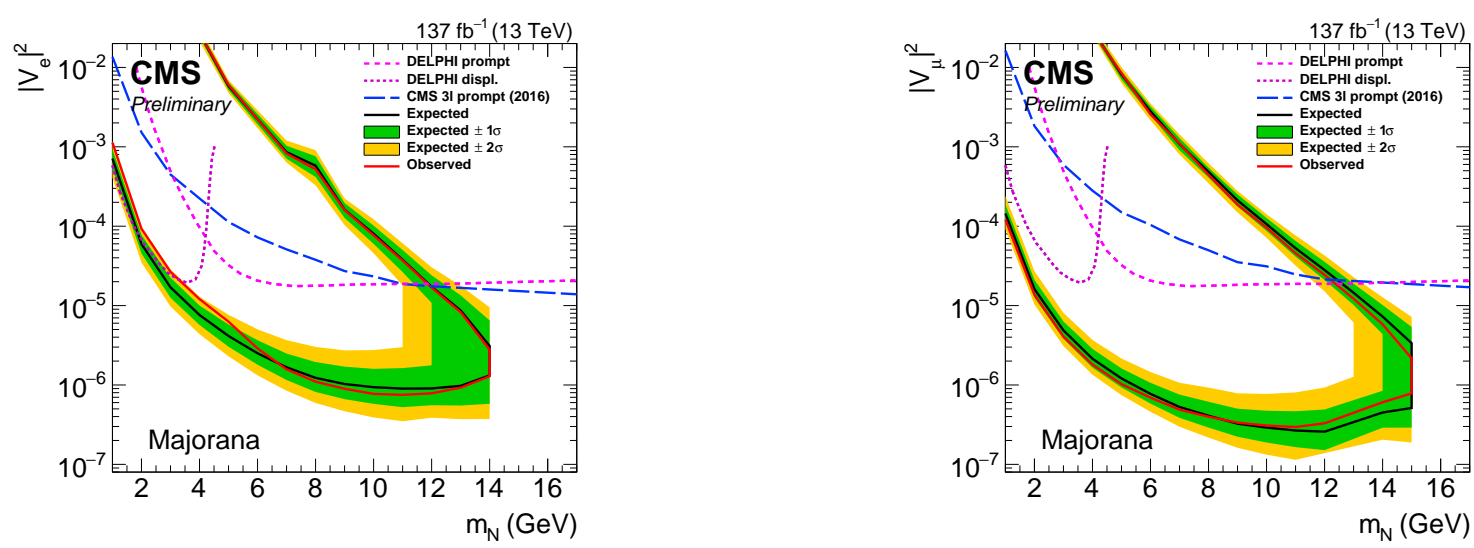

Figure 9: Limits on $\left|V_{N e}\right|^{2}$ (left) and $\left|V_{N \mu}\right|^{2}$ (right) as a function of $m_{N}$ for a Majorana HNL. Results from the Delphi and the CMS Collaborations are shown for reference.

\section{Run 3 opportunities for Physics}

In Run 3 CMS is expected to collect an integrated luminosity at least equal to that collected in Run 2. and most likely larger. All analyses will be repeated with at least twice the integrated luminosity; they will also benefit of additional improvements coming from further developments of the analysis methods, including ML techniques; and they will aim at further reduction of systematic uncertainties.

Furthermore, we are working on the development of new triggers, especially addressed at long lived particles (LLP), and on the further improvements of the non-conventional data taking methods: Data Scouting and (B-)parking. During the last few years, we have developed an heterogeneous event reconstruction using CPUs and GPUs that will be deployed in the HLT for Run 3 and we are already planning to offload at least $30 \%$ of the reconstruction to the GPUs. More specifically, the ECAL and HCAL local reconstruction and the pixel reconstruction and tracking have already been ported to GPUs.

\subsection{LLP improvements}

A lot of activity is ongoing towards new developments in the Level 1 trigger (L1) with the aim to increase efficiency for displaced signatures. We have increased the efficiency for displaced muons using the Kalman Filter at L1, we are planning to extend muon triggers to hadronic showers in the muon chambers that could be originated by long lived hadrons decaying. We are also exploring the possibility to exploit ECAL and HCAL timing information and HCAL depth information to increase the trigger efficiency for LLPs. A the same time corresponding developments are ongoing for the HLT.

\subsection{Scouting}

Scouting consists in analyzing the data using the online reconstruction in the HLT farm and saving it offline with a reduced data format (do not save the full event data). In Run 2 the scouting analysis was based on approximately $5 \mathrm{kHz}$ of triggers of which approximately $1 \mathrm{kHz}$ had the full 
particle flow reconstruction. For Run 3 we aim at running particle flow on a much larger rate, possible adding additional L1 triggers, thanks to an extended usage of pixel tracks and to the GPU reconstruction.

\subsection{B-parking}

In 2018 we used low $p_{T}$ displaced muon triggers to record a sample of unbiased B hadron decays recoiling against the triggered muon. The average parked trigger rate of approximately 2 $\mathrm{kHz}$ was reconstructed after the end of the run. This data sample enables several analyses on LFU violation that are currently in progress. In view of Run 3, we are studying how to further optimize the B-parking trigger and in particular we are evaluating the possibility in enriching the sample in di-electron decays.

\section{CMS Phase II upgrades for HL-LHC}

After Run 3, in 2027, the LHC will start its high luminosity phase that is expected to provide ATLAS and CMS with 3000 to $4000 \mathrm{fb}^{-1}$. A comprehensive review of the physics at the HL-LHC is given here [12]. CMS is currently extending and updating these studies in view of the Snowmass workshop and the projections of the sensitivity to the measurement's of the $W^{ \pm} W^{ \pm}$and $W Z$ Vector Boson Scattering processes [13] are the first of these results. The sensitivity to the contributions of $W_{L}^{ \pm} W_{L}^{ \pm}, W_{L}^{ \pm} W_{T}^{ \pm}$, and $W_{T}^{ \pm} W_{T}^{ \pm}$, measured in the $W^{ \pm} W^{ \pm}$or in the initial-state parton-parton center-ofmass reference frame is extrapolated from full Run 2 analysis and it is shown in Fig. 10 in terms of uncertainty and discovery sensitivity.
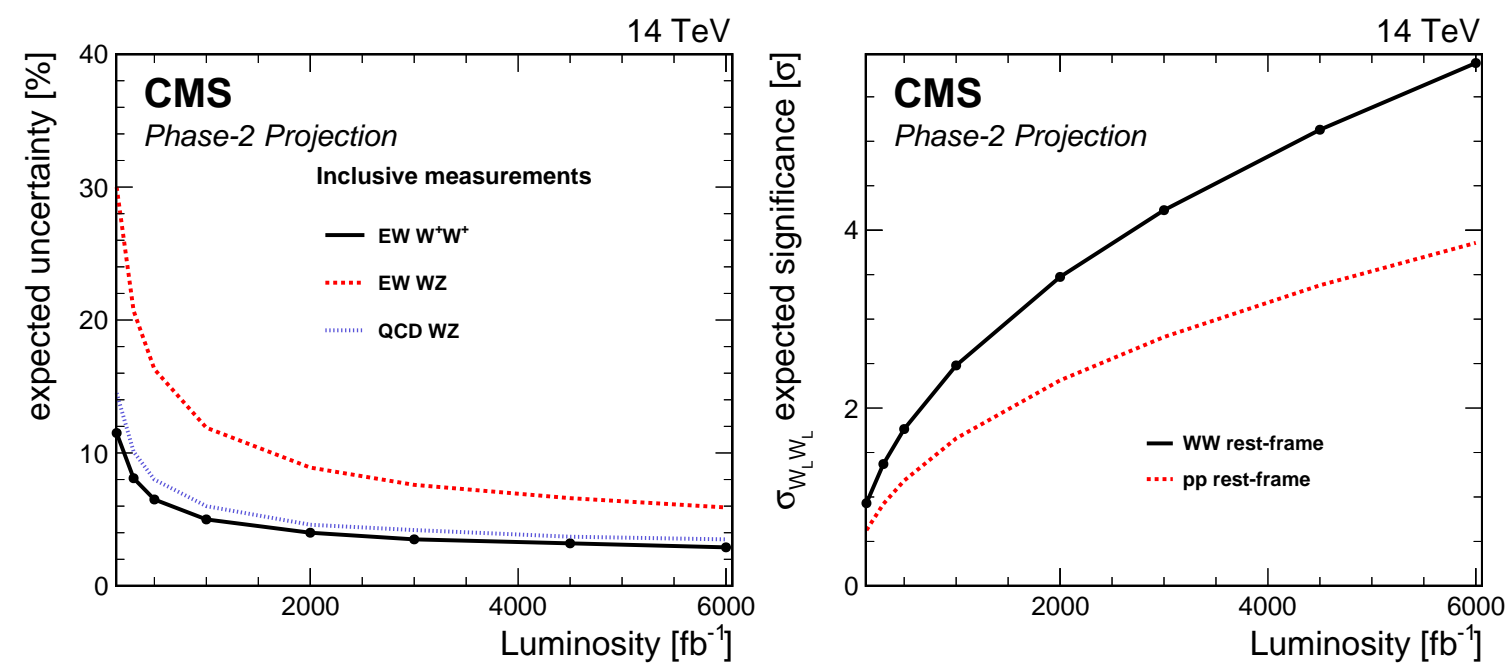

Figure 10: (left) Projected estimated uncertainty in the EW $W^{ \pm} W^{ \pm}, \mathrm{EW} W Z$, and QCD $W Z$, cross section measurements as a function of the integrated luminosity. (right) Projected estimated significance for the EW $W_{L}^{ \pm} W_{L}^{ \pm}$process as a function of the integrated luminosity for the $W^{ \pm} W^{ \pm}$and parton-parton center-of-mass reference frames. 


\section{Summary}

The current activities and results from the CMS experiment are reviewed. Some of the most recent results from the analysis of the Run 2 data are presented, including detector related performance studies and physics analyses. The preparation for Run 3 is also addressed and the prospects for the High Luminosity phase of the LHC are briefly outlined.

\section{Acknowledgments}

I would like to thank the LHC team which made all this possible in Run 2 an for all the activities they are performing to lead us to a Run 3 and beyond.

\section{References}

[1] CMS Collaboration, JINST 3 (2008), S08004 doi:10.1088/1748-0221/3/08/S08004

[2] CMS Collaboration, Eur. Phys. J. C 81 (2021) 800 doi:10.1140/epjc/s10052-021-09538-2 [arXiv:2104.01927 [hep-ex]].

[3] CMS Collaboration, CMS-PAS-LUM-17-001, https://cds.cern.ch/record/2257069.

[4] CMS Collaboration, CMS-PAS-SMP-18-014, https://cds.cern.ch/record/2775364.

[5] CMS Collaboration, CMS-PAS-BPH-21-004, https://cds.cern.ch/record/2776769.

[6] CMS Collaboration, JHEP 01 (2021), 148 doi:10.1007/JHEP01(2021)148 [arXiv:2009.04363 [hep-ex]].

[7] CMS Collaboration, CMS-PAS-HIN-20-004, https://cds.cern.ch/record/2767048.

[8] CMS Collaboration, CMS-PAS-B2G-21-002, https://cds.cern.ch/record/2776919.

[9] CMS Collaboration, CMS-PAS-B2G-20-001, https://cds.cern.ch/record/2759857.

[10] [CMS], CMS-PAS-EXO-20-009, https://cds.cern.ch/record/2777047.

[11] [CMS], CMS-PAS-SUS-20-004, https://cds.cern.ch/record/2777125.

[12] A. Dainese, M. Mangano, A. B. Meyer, A. Nisati, G. Salam and M. A. Vesterinen, doi:10.23731/CYRM-2019-007

[13] CMS Collaboration, CMS-PAS-FTR-21-001, https://cds.cern.ch/record/2776773. 